\title{
Design of a intellectualized ultrasonic generator
}

\author{
Xiyang Liu ${ }^{1}$, Xiaodong Cui ${ }^{2}$, Fuhua $\mathrm{Ma}^{3}$ \\ ${ }^{1}$ College of Information Science and Technology, Hainan University, Hainan 570228, China \\ ${ }^{2}$ Science and Technology Department of Hainan province, Haikou 570203, China \\ ${ }^{3}$ School of Information Science and Engineering, Guangxi University For Nationalities, Nanning, \\ 530006, China
}

Keywords: ultrasonic; frequency tracking; single chip microcomputer

Abstract. The structure, operation principle and realizable method of a ultrasonic generator are introduced in this paper. It can realize the best frequency search. Output power can be adjusted.

\section{Introduction}

Power ultrasonic technology has been widely applied in various aspects of chemistry ${ }^{[1]}$. Power ultrasound can accelerate and control chemical reaction, improve reaction yield, reduce reaction condition and cause new chemical reaction ${ }^{[2]}$. It is of unique efficiency in chemical reaction ${ }^{[3]}$. Power ultrasound has already become a new research hotspot. However, most existing ultrasonic excitation devices can only be used for excitation of transducer in single model within a narrow range. Besides, it is very difficult for power to reach continuous adjustment from 0 to full load. To meet experimental requirements, we designed and developed high-efficiency ultrasonic excitation source with very strong universality and continuously controllable broadband and output power. This excitation source can produce ultrasonic drive signals between $20 \mathrm{KHZ}$ and 500KHZ. The maximum output power can reach $500 \mathrm{~W}$, realizing automatic searching of the optimum resonant frequency between transducer and series inductance, and providing convenience for replacement of transducer in different models.

For monitoring of environmental pollutants, it is generally necessary for treatment of environmental samples, so as to separate target objects from matrixes and make it convenient for separation detection in the next stage. For traditional and conventional methods, considerable toxic organic reagents need to be used, with tedious procedures and large workload. In recent years, therefore, many methods are proposed for improvement. We have successfully applied the researched excitation source into marked soil samples (the method of ultrasonic-assisted extraction ${ }^{[4]}$ is adopted). The application turns out to be of good effects.

\section{Circuit composition of system hardware}

The system is composed of single chip microcomputer AT89S52, ultrasonic frequency signal generator, numerical control DC power supply, resonance matching detection and power amplification output. Schematic diagram of the system is shown in Fig. 1.

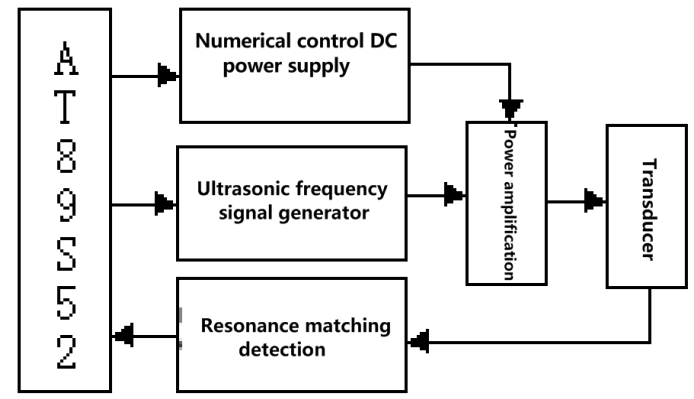

\subsection{Ultrasonic frequency signal generator}

Fig. 1 Schematic Diagram of the System

Ultrasonic frequency signal generator will produce an ultrasonic frequency voltage signal as 
signal source of power amplification circuit in the next stage. In this paper, MAX038 high-frequency precision waveform generator is adopted as signal source. This generator can produce sine wave and several waveforms between $0.1 \mathrm{~Hz}$ and $20 \mathrm{MHz}$, with the maximum frequency as high as 40MHz. Through control over levels of input pins A0 and A1 of MAX038, rectangular wave, triangular wave and sine wave can be generated respectively. When ends of A0 and $\mathrm{A} 1$ are placed at 01 , sine wave can be generated. When ends of A0 and A1 are placed at 00, rectangular wave can be generated. When ends of $\mathrm{A} 0$ and $\mathrm{A} 1$ are placed at 10 , triangular wave can be generated. Rectangular wave is adopted as waveform of this system. A0 and A1 are directly placed at 00, as shown in Fig. 2.

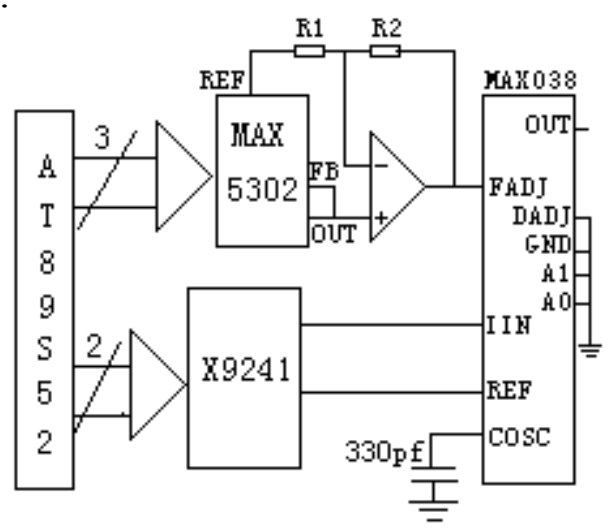

Fig. 2 Schematic Diagram of Ultrasonic Frequency Signal Generator

After the determination of the value of $\mathrm{C}_{\mathrm{f}}$, output frequency of MAX038 is mainly controlled by input current at IIN end, but it is affected by voltage at FADJ and DADJ ends. For control over current $\mathrm{I}_{\mathrm{IN}}$, digital potentiometer X9241 is externally connected between the two pins: IIN and REF. According to the requirements of output frequency between $20 \mathrm{KH}_{\mathrm{Z}}$ and $500 \mathrm{KH}_{\mathrm{Z}}, \mathrm{C}_{\mathrm{f}}$ is designed as $330 \mathrm{PF}$, which can cover the frequency range of $10 \mathrm{KHZ}-1 \mathrm{MHZ}$. The range of frequency is large enough. After the determination of the value of $\mathrm{C}_{\mathrm{f}}, \mathrm{I}_{\mathrm{IN}}$ can be adjusted through adjustment to the value of digital potentiometer, so as to adjust center frequency of MAX038 and thus make fine adjustment to output frequency through 12-digit D/A convertor MAX5302 connected to Pin FADJ of MAX038. 12-digit D/A convertor divides the frequency range of $20 \mathrm{~K}-500 \mathrm{KHz}$ into small enough frequency intervals. These small frequency intervals are used for scanning search of resonant frequency of transducer and series matching inductance. In actual use, it can allow transducer to work in the optimum resonance state.

\subsection{Power amplification circuit}

Push-pull, semi-bridge, full-bridge and other patterns can be adopted for ultrasonic power amplification circuit. The voltage is not high on full-bridge circuit switch tube, which is applicable to relatively large power output. The maximum output power of ultrasonic system researched in this paper is 500W. Full- bridge circuit is selected. For switch tube, IRFP460 produced by IR Company is selected as power amplifier. To realize controllable output power, two plans are generally used at present. In the first plant, turn-on time ratio of switch tube is changed by changing duty ratio of rectangular wave, so as to adjust output power. In the second plan, amplitude of AC output voltage is changed by changing DC voltage on full-bridge circuit, so as to adjust the output power. Upon comparison, the second plan is of simple drive circuit, and it is easier to realize adjustment to output power from 0 to full load. Therefore, the second plan is adopted in this paper. Fig. 3 shows the schematic diagram. 


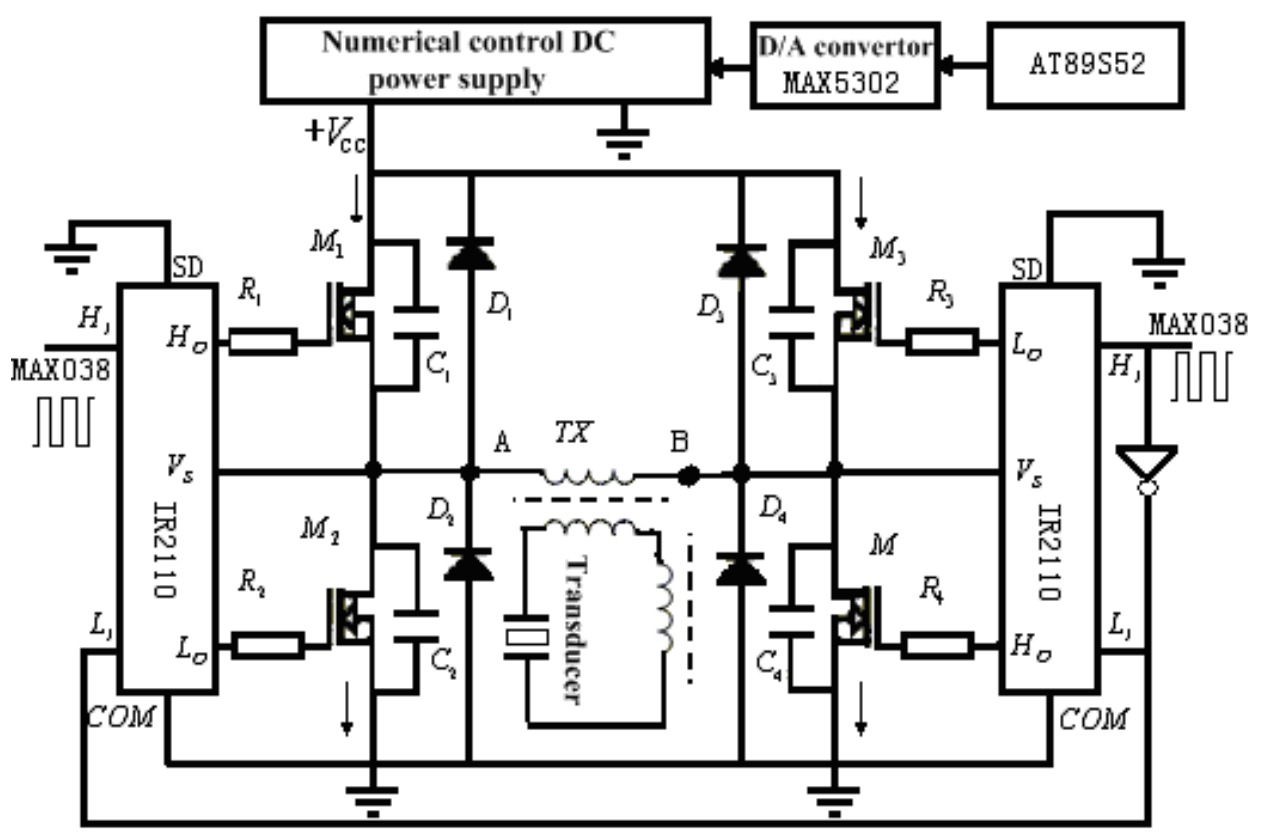

Fig 3. Schematic Diagram of Power Amplification Circuit

Exclusive driver chip IR2110 is adopted as drive of full-bridge power amplification circuit, with transformer coupling output. In Fig. $3, D_{1}, D_{2}, D_{3}$ and $D_{4}$ are fast recovery diodes. $C_{1}, C_{2}, C_{3}$ and $C_{4}$ stand for output capacitance of field efficiency tube. Square wave excitation mode is adopted for full-bridge circuit. Under the excitation of square wave, full bridge is conductive for all tubes at diagonal lines at the same time. Working sequence of MOSFET is shown as follows: $\mathrm{M}_{1}$ and $\mathrm{M}_{4}$ are conductive first. At the same time, $\mathrm{M}_{2}$ and $\mathrm{M}_{3}$ are cut off, followed by $\mathrm{M}_{1}$ and $\mathrm{M}_{4}$. At the same time, $\mathrm{M}_{2}$ and $\mathrm{M}_{3}$ are conductive, or opened or closed in the opposite sequence. During power adjustment, AT89S52 is used to adjust input voltage of numerical control DC power supply through D/A convertor, making it to change between $0 \mathrm{~V}$ and $5 \mathrm{~V}$, so as to drive changes of output voltage $\mathrm{V}_{\mathrm{CC}}$, achieve the purpose of changing amplitude of current voltage on transformer, and thus control output power of ultrasonic wave.

\subsection{Resonance matching detection}

During operation of ultrasonic transducer, it should be operated under resonance state. Or, the conversion efficiency is low from electric energy and mechanical energy. At the same time, the service life of devices will be affected as well. Resonant frequency of ultrasonic transducer is mainly decided by its features and load characteristics. However, this resonant frequency will be also affected by load and a series of factors. Drifting will be produced. Therefore, real-time detection for operation of transducer under resonance state and realization of automatic tracking of automatic tracking are of great importance in ultrasonic devices.

While connected to circuit for operation, transducer must be provided with corresponding matching network. The most common method is to use single inductance for its matching. Single inductance matching includes two patterns: series connection and parallel connection. However, active resistance of ultrasonic system has no change after shunt inductance. At the same time, this pattern lacks filter action. However, series inductance can reduce active resistance and give consideration to tune and filter action at the same time. Therefore, series inductance matching is adopted. Transducer before and after series inductance are analyzed by sweeper. Fig. 4 shows its conductivity response curve. 


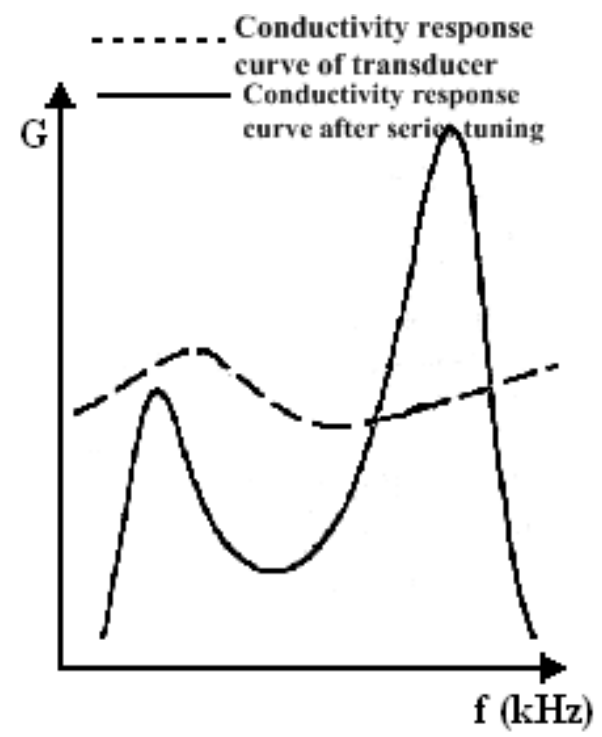

Fig. 4 Conductivity Response Curve of Transducer after Series Tuning

As shown in Fig. 4, the conductivity of ultrasonic transducer has two peak values after series inductance. The latter peak value is caused by impedance change due to addition of inductance. It is suitable for work of transducer at this frequency. In this system, load is first subject to frequency sweeping once after startup. The frequency at the two maximum peak values is judged by detecting and comparing effective values of transducer current, so as to take the smaller frequency as datum resonant frequency of the system. Due to dynamic influence of load, however, actual resonant frequency will fluctuate in the operating process. Within a certain range around datum frequency, therefore, automatic tracking of transducer resonant frequency can be realized by adjusting the frequency of excitation source with single chip microcomputer to maximize output current of the system. In this system, current value is measured by connecting in series a $0.5 \Omega$ sample resistance between transducer and the ground and then collecting current signal of the transducer. This signal is converted into true virtual value via AC signal/ effective value conversion chip. Then, the signal is sampled by A/D collection chip MAX199, and then sent to single chip microcomputer for comparison and processing, so as to determine whether output frequency reaches resonance requirements of the transducer, as shown in Fig. 5.

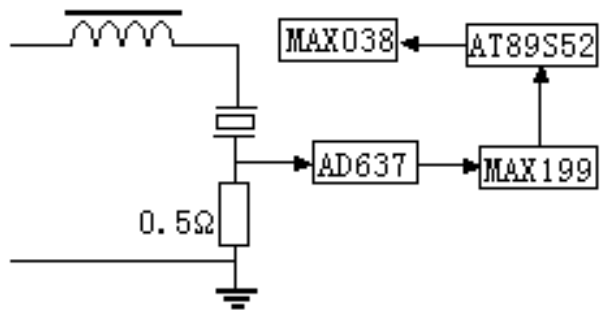

Fig. 5 Resonance Detection Signal Collection Circuit

\section{System software design}

The goal of system software design is to make ultrasonic system to work under resonance state according to present power. In the realization process, drifting of resonant frequency is judged by the system through detecting changes of current. If drifting exists, the operating frequency will be adjusted to a new resonant frequency. The whole system procedure includes main program, resonant frequency search subprogram and power control subprogram. The purpose of power control subprogram is to make the system to work according to preset output power, including the design on man-machine interaction program. Fig. 6 shows the process of resonant frequency search subprogram.

In the frequency search process, 12-digit D/A convertor is used to divide the range of output frequency of MAX038 into frequency intervals $(\Delta \mathrm{f})$ small enough. Due to the existence of $\Delta \mathrm{f}$, however, the maximum current frequency searched may be slightly less than or greater than series 
resonance frequency. At this moment, the error caused is very small. In projects, they are deemed as equivalent to each other.

\section{Conclusion}

In control circuit of ultrasonic excitation system, single chip microcomputer is used to control relevant parameters, realizing automatic tracking of resonant frequency and continuous adjustment to output power between 0 and full load. According to the use in chemical experiment, the system is of simple and convenient operation, compact structure, and strong real-time tracking of frequency, properly meeting experimental requirements.

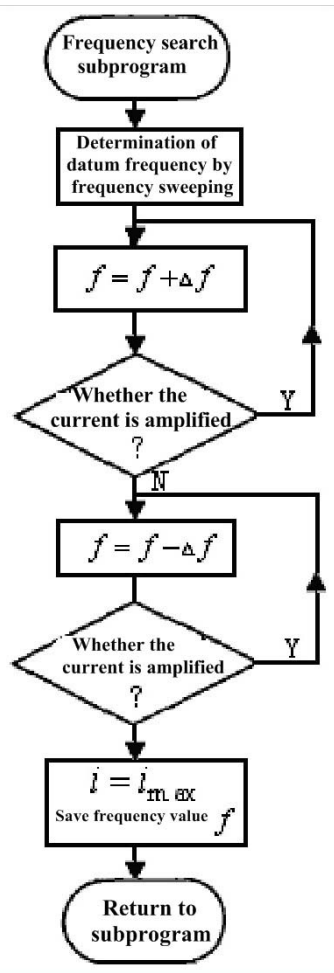

Fig. 6 Flowchart of Frequency search subprogram

\section{Acknowledgments}

This paper is: 1. education and teaching research project of Hainan University: "Research on Application of PBL Teaching Method in Teaching of Electronic Information Courses" (No.: hdjy1330); and 2. scientific research in colleges and universities funded by the Education Department of Hainan Province: "Program Control Ultrasonic Experiment System" (No.: Hj2010-03)

\section{References}

[1] Li Jida and Zang Hongjun, New Progress of Ultrasonic Wave Applied in Organic Synthesis, Journal of Hebei University (Natural Science Edition), 2000, Vol. 20 (1);

[2] Li Shuyu, Research Status of Power Ultrasonic Technology and Its New Progress, Journal of Shaanxi Normal University (Natural Science Edition), 2001, Vol. 29 (1);

[3] Chief editor: Yuan Yiquan, Modern Ultrasonic Theory and Application: Section 2 of Chapter 8, Nanjing University Press, 1996. 